\title{
RESEARCH HIGHLIGHT Expanding cultural and ancestral representation in psychiatric genetic studies
}

\author{
Jooeun Kang (iD) and Douglas M. Ruderfer ${ }^{1,2,3}$ \\ Neuropsychopharmacology (2020) 45:1593-1594; https://doi.org/10.1038/s41386-020-0676-1
}

The vast majority of samples included in genome-wide association studies (GWAS) have been of European descent [1]. While the proportion of non-European samples has increased over the last 10 years, this has been predominantly driven by the increase in studies of local populations in Asia [2]. Much of the world remains largely unrepresented in genetic studies of common traits and diseases that affect individuals globally. The lack of ancestral diversity limits the potential to identify relevant genetic variation that may exist at different frequencies or effect sizes in nonEuropean populations. Further, when considering potential clinical utility of genetic information, such as recent consideration of using polygenic risk scores (PRSs), there exists substantial evidence that disparities will result from poor translatability across populations [3]. Previous work in psychiatric disorders, specifically schizophrenia, has demonstrated value and improvement when using PRSs built and applied in samples with shared ancestry [4].

Hispanic, Latino, and native populations from South America are a few of the ancestral groups that remain severely understudied. In this issue, Shen et al. perform the largest current GWAS of psychiatric traits in a South American sample $(n=3308)$, expanding genetic research to include this underrepresented population. They recruited women who received their prenatal care from the Instituto Nacional Maternal Perinatal in Lima, Peru as part of the Pregnancy Outcomes, Maternal and Infant Study (PrOMIS cohort). The investigators used GWAS summary statistics of depression and posttraumatic stress disorder (PTSD) generated from predominantly European ancestries as the discovery datasets and the PrOMIS cohort as the target sample to test PRSs with depression, PTSD, and suicidal ideation/self-harm. The PRSs showed limited but significant explanation of phenotypic variance of each trait: explaining up to $0.62 \%$ of the phenotypic variance of depressive symptoms, and $0.3 \%$ for the phenotypic variance of PTSD and suicidal ideation/self-harm. As expected, these cross-ancestral PRSs explained less phenotypic variance than those generated from the same discovery GWAS but applied to target samples of European ancestries. The authors also performed GWAS for depression, PTSD and suicidal ideation or selfharm in the PrOMIS cohort. These were underpowered to identify genome-wide significant loci, but the summary statistics have been provided to the genetics community for inclusion into other GWAS efforts on these phenotypes for improved diversity.

Of particular importance, these patients represent a high-risk population of South American women of reproductive age and lower socioeconomic status. More than a third experienced intimate partner violence, nearly three quarters of the participants have a history of child abuse and about half of the participants expressed hardship in accessing basic foods. This represents significantly different environmental conditions to those expected among the majority of patients recruited from wealthier countries in genetic studies of psychiatric conditions to date. Not surprisingly, the PrOMIS cohort had increased prevalence and severity of psychiatric phenotypes including a mean PHQ-9 depression score of 8.2 , a mean PCL-C PTSD score of 27.4 and a $15.4 \%$ prevalence of suicidal ideation/self-harm. Demonstrating that European derived PRSs are significant in this population is important for showing that aggregate genetic risk is shared across varying populations, environmental, and cultural conditions. The degree to which the reduction in significance of PRS is a product of these differing factors remains unknown. This question is of utmost importance and samples like the one used here will be crucial in addressing it.

If the goal is to use PRS clinically, it is important that their utility is not a product of patient ancestry which would result in worse outcomes among certain populations. This work demonstrates that PRSs derived from GWASs of largely European ancestries and applied to a high-risk South American sample significantly correlate with psychiatric outcomes. However, these results are less significant than if performed within a European sample, showing potential for disparity. Importantly, clinical utility will be based on predictive performance assessed at the level of individual risk and not at the level of the population as done here and in most PRS-based analyses. Therefore, it is more appropriate to interpret these results as associations of genetic risk with phenotypes than true predictions. Whether PRS will provide clinical utility for psychiatric traits remains to be seen.

This study highlights two gaps in the field of psychiatric genetic research: underrepresentation of individuals from non-European ancestries and limited capture of relevant environmental factors. Continued efforts to increase representation of marginalized populations in psychiatric genetic studies will benefit the field by helping elucidate the relationship between environmental effects and genetics on behavioral health. The study also delineates important directions for future research involving trans-ancestry samples, namely comparison of findings to existing European-only studies to better understand the effect of ancestral differences in interpreting the potential implications of genetic study findings. Most importantly, these efforts will be instrumental in understanding and potentially reducing mental health disparities across populations of differing ethnicities and socioeconomic backgrounds.

\footnotetext{
${ }^{1}$ Division of Genetic Medicine, Department of Medicine, Vanderbilt Genetics Institute, Vanderbilt University Medical Center, Nashville, TN, USA; ${ }^{2}$ Department of Biomedical

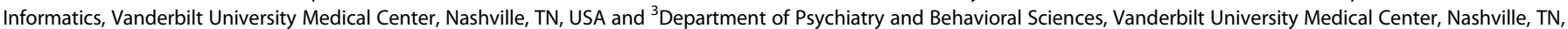
USA

Correspondence: Douglas M. Ruderfer (douglas.ruderfer@vanderbilt.edu)
}

Received: 18 February 2020 Revised: 24 March 2020 Accepted: 6 April 2020

Published online: 15 April 2020 


\section{FUNDING AND DISCLOSURE}

This work was supported by NIMH grants R01MH111776, R01MH116269, and R01MH121455. The authors have no financial conflicts of interest to disclose

\section{AUTHOR CONTRIBUTIONS}

JK and DMR contributed equally to the conception, drafting, and revising of this work.

\section{ADDITIONAL INFORMATION}

Publisher's note Springer Nature remains neutral with regard to jurisdictional claims in published maps and institutional affiliations.

\section{REFERENCES}

1. Need AC, Goldstein DB. Next generation disparities in human genomics: concerns and remedies. Trends Genet. 2009;25:489-94.

2. Popejoy AB, Fullerton SM. Genomics is failing on diversity. Nature. 2016; 538:161.

3. Martin AR, Kanai M, Kamatani Y, Okada Y, Neale BM, Daly MJ. Clinical use of current polygenic risk scores may exacerbate health disparities. Nat Genet. 2019; 51:584.

4. Bigdeli TB, Genovese G, Georgakopoulos P, Meyers JL, Peterson RE, lyegbe CO, et al. Contributions of common genetic variants to risk of schizophrenia among individuals of African and Latino ancestry. Mol Psychiatry. 2019;1-13. https://doi. org/10.1038/s41380-019-0517-y. 